IdeAs

Idées d'Amériques

16 | 2020

Les marges créatrices : intellectuel.le.s afrodescendant.e.s et indigènes auX Amériques, XIX-XXe siècle

\title{
Citoyenneté économique et citoyenneté politique des femmes aux Etats-Unis
}

Jeanne Boiteux

\section{OpenEdition \\ Journals}

Édition électronique

URL : http://journals.openedition.org/ideas/9618

DOI : 10.4000/ideas.9618

ISSN : 1950-5701

Éditeur

Institut des Amériques

Référence électronique

Jeanne Boiteux, «Citoyenneté économique et citoyenneté politique des femmes aux Etats-Unis », IdeAs [En ligne], 16 | 2020, mis en ligne le 01 octobre 2020, consulté le 10 décembre 2020. URL : http:// journals.openedition.org/ideas/9618; DOI : https://doi.org/10.4000/ideas.9618

Ce document a été généré automatiquement le 10 décembre 2020.

\section{(c) (1) $\Theta \Theta$}

IdeAs - Idées d'Amériques est mis à disposition selon les termes de la licence Creative Commons Attribution - Pas d'Utilisation Commerciale - Pas de Modification 4.0 International. 


\title{
Citoyenneté économique et citoyenneté politique des femmes aux Etats-Unis
}

\author{
Jeanne Boiteux
}

1 Si les célébrations de la ratification du $19^{e}$ amendement de la Constitution étatsunienne affirment davantage que par le passé que 1920 fut surtout une victoire pour les femmes blanches (Jones M., 2020), il reste à souligner la nécessaire continuité des combats menés pour les droits économiques des Américaines et notamment ceux des femmes de couleur. Distincte de la citoyenneté politique, la citoyenneté « économique » est aujourd'hui un objet d'étude qui complexifie notre vision des luttes féministes.

\section{Un concept récent et multiforme}

2 La citoyenneté économique est un outil pour l'étude de la relation de l'individu au corps social. Sa définition varie selon les auteurs et les disciplines - histoire, sociologie et même anthropologie - mais toutes ses acceptions sont significatives pour le champ de l'histoire des femmes et du genre. Pour les chercheurs et chercheuses que ce concept a intéressés, l'accès à l'emploi et l'égalité salariale sont des enjeux aussi centraux pour la définition des femmes en tant que citoyennes que leur capacité à se rendre aux urnes.

3 La citoyenneté économique peut être vue comme un prolongement de la citoyenneté sociale décrite par T. H. Marshall. Depuis ses travaux dans le champ de l'étude de la citoyenneté, il est admis que le concept de citoyenneté recouvre aussi bien des aspects civils et politiques que sociaux. Ainsi qu'il le décrit, la naissance de l' « Etat providence " dans plusieurs pays européens au $\mathrm{xx}^{\mathrm{e}}$ siècle met en lien appartenance à la communauté nationale et droit aux aides sociales de l'Etat. Marshall définit la citoyenneté comme « $[u] n$ statut qui est conféré à ceux qui sont membres de plein droit d'une communauté. Tous ceux qui jouissent de ce statut sont égaux en droits et en devoirs s'y référant » (Marshall T.H., 1950 : 28-29). Dans ce cadre, les droits associés à la 
citoyenneté permettraient un accès à certaines ressources. Après Marshall, d'autres auteurs ont continué à explorer l'idée qu'il pouvait s'agir de garanties concernant l'accès au marché du travail.

4 L'historienne états-unienne Alice Kessler-Harris s'est intéressée à l'accès des femmes et des minorités états-uniennes au marché du travail. Aux Etats-Unis, le travail comme source de revenus sous-tend l'appartenance à la communauté civile et politique; le statut de salarié donne droit à de nombreux avantages sociaux. Kessler-Harris définit la citoyenneté économique comme un ensemble de droits, notamment celui de « choisir son travail » et de toucher une rémunération correcte pour ce dernier, sans oublier le droit d'accéder aux formations facilitant l'accès au marché du travail (Guillen A., 2003 : 186-195). L'Américaine du début du Xxe siècle, si elle ne disposait pas du suffrage dans la plupart des Etats, devait également subir des discriminations dans l'enseignement technique et supérieur et sur le lieu de travail, la médiocrité des salaires féminins et la méfiance extrême des syndicats masculins à l'égard des revendications des ouvrières.

D'autres auteurs et autrices mettent en avant une autre facette de l'indépendance économique : non plus les conditions qui la garantissent, mais ce qu'elle rend possible, c'est-à-dire l'acte d'achat. Aux Etats-Unis, le pouvoir d'achat est l'ingrédient essentiel de la nouvelle identité citoyenne dont les contours sont progressivement dessinés par l'avènement d'une société de consommation au cours du $\mathrm{xx}^{\mathrm{e}}$ siècle. Est citoyen.ne non seulement qui vote, mais aussi qui est capable de consommer selon certains paramètres. Le nouveau citoyen-consommateur a l'oreille du gouvernement aussi bien en tant que citoyen qu'en tant que consommateur : lorsque le président Franklin Delano Roosevelt supervise dans les années 1930 la création de structures pour encadrer les prix et représenter les consommateurs auprès des entreprises, son gouvernement légitime les intérêts des consommateurs comme intérêts citoyens. Des organisations de consommatrices, par leur ferveur, suscitèrent même des comparaisons avec le mouvement pour le suffrage (Jacobs M., 2005 : 2-6, 97-98, 104). Ce nouveau pouvoir renforce certaines formes d'action politique. La possibilité de bénéficier des justes revenus de son activité salariale ou entrepreneuriale et celle de dépenser cet argent, considérées ensemble, forment la base du consumer activism, une tradition politique émergente au XIXe siècle, et qui se fonde sur l'utilisation d'un pouvoir d'achat collectif comme acte politique. L'identité du travailleur rémunéré et celle du consommateur sont prises comme identités politiques et ouvrent la porte à une forme de reconnaissance sociale (Glickman L., 2009 : 25, 155).

\section{Reconnaissance sociale de l'emploi aux Etats-Unis}

Le problème de l'accès à l'emploi se pose de manière plus aiguë pour les femmes, dont la place sur le marché du travail et l'accès aux «bons » emplois - les plus stables et les plus rémunérateurs - sont moins assurés. Les avantages sociaux procurés par l'accès à l'emploi se révèlent d'ordre symbolique autant que matériel - assurances, santé, retraite - et ce de manière croissante depuis la présidence de Bill Clinton.

7 On peut retracer loin les origines du concept de workfare. Dès le $\mathrm{xvI}^{\mathrm{e}}$ siècle, la réforme des Poor Laws et sa diabolisation de l'oisiveté constituent un nouveau modèle social, exporté en Nouvelle-Angleterre et renforcé par les prescriptions de l'éthique protestante. L'importance de ce système de valeurs ne diminuera guère avec l'indépendance des colonies britanniques, d'autant plus en l'absence de définition 
légale de la citoyenneté. Pour les Pères Fondateurs imprégnés des idées des Lumières, la liberté est la valeur première qui fait l'individu. Est libre celui qui possède son propre corps et jouit de son labeur; le droit de propriété découle nécessairement de cette propriété originelle. Dans une société d'individus autonomes passant contrat entre eux, pas de place pour les esclavisé.e.s, hommes et femmes qui ne possédaient pas leur propre corps, et qui par là ne pouvaient prétendre à aucune liberté individuelle. Plus largement, toute femme, même libre, même blanche, était à cette époque définie comme dépendante, puisque le corps de la femme mariée était propriété de son mari (Daune-Richard A.M., $2008:$ 39-50).

8 La Constitution des Etats-Unis ne définit pas clairement la citoyenneté ; le droit de vote, souvent utilisé comme son indicateur, n'était à l'origine considéré que comme un privilège accordé à certains citoyens. En outre, sa délimitation relève de la compétence des Etats. Cette absence de clarté fut la cause de débats houleux lors de l'avènement du suffrage dit « universel» (masculin, blanc) suite à la suppression des restrictions censitaires. Le bon sens voulait que, si blanches et nées aux Etats-Unis, les femmes fussent des citoyennes, mais l'examen minutieux de leurs droits révélait l'étendue de leur impuissance juridique et politique. Pas d'égalité de droits et de devoirs pour les femmes, que l'on ne voyait pas davantage comme des soldats que comme des productrices, mais une citoyenneté tronquée.

9 Leur identité économique constituait un obstacle. Si la citoyenneté américaine n'avait pas de définition claire, il existait bien un idéal: l'idéal jeffersonien du petit producteur-citoyen. La propriété et l'indépendance économique étaient conçues comme la garantie de qualités morales jugées nécessaires pour la jouissance des droits et l'exercice des devoirs du citoyen. Celui-ci était défini à la fois positivement et négativement selon des termes économiques : c'était l'homme capable d'assurer seul sa subsistance, qui ne peut subir des pressions de la part d'un patron ou d'un mécène, et peut donc faire un libre exercice de sa raison lors de sa participation aux affaires de la cité. C'était encore le propriétaire, que l'inquiétude pour ses biens pousse à rechercher la prospérité commune.

10 La condition féminine est alors marquée par l'absence d'une telle indépendance. On considère que les intérêts politiques des femmes sont définis par l'identité sociale du breadwinner, théoriquement source principale des revenus de la famille. De plus, concrètement, les femmes étaient nombreuses à dépendre de leur mari pour leur subsistance. Cet entrelacement étroit de l'indépendance économique et de l'indépendance politique a donné lieu à l'éclosion de revendications spécifiques au contexte états-unien. A la convention pour les droits des femmes de Worcester, en 1850, on exposait l'idée que les femmes célibataires qui payaient des impôts remplissaient les conditions morales nécessaires pour bénéficier du suffrage. La notion d'égale représentation était aussi utilisée pour exiger un droit à l'éducation supérieure, et notamment aux études de médecine (Isenberg N., 1998 : 35-36).

11 Après la guerre de Sécession en 1865 , ces critiques émanèrent de mouvements utopistes et coopératifs ainsi que des milieux ouvriers féminins. Lara Vapnek a étudié les demandes de certaines ouvrières en marge du mouvement pour le suffrage féminin et affirme qu'à New York, l'intérêt des ouvrières pour le vote précéda celui des femmes des classes moyenne et supérieure. Elles qui étaient la principale source de revenus de leur foyer utilisèrent l'existence de personnes à leur charge comme un argument pour demander le suffrage. Ce n'était pas leur seul horizon et leurs stratégies étaient 
multiples; il pouvait s'agir de faire reconnaître à l'Etat sa responsabilité sociale vis-àvis des femmes autant que d'affirmer un droit à l'éducation et à une juste rémunération (Vapnek L., $2009: 1,7,12,20,130-131$ ).

12 Les aides sociales créées par le New Deal dans les années 1930 ne furent pas octroyées aux femmes en tant que salariées, mais en tant que mères ou veuves, dans une société de crise où l'on ne tolérait plus le travail salarié des femmes mariées. Les gains du New Deal étaient limités pour les Américaines et pour les Noirs américains. Pour cette raison, de manière particulièrement vive dans les années 1950 et 1960, les femmes de couleur au sein de plusieurs mouvements pour les droits civiques continuèrent de porter des revendications d'ordre à la fois politique et économique, en exigeant un accès à des champs aussi divers que le processus électoral, le marché du travail, les programmes d'aide fédérale et le marché de l'immobilier. Elles se donnaient pour but de redéfinir en les élargissant les droits associés à la citoyenneté. En particulier, les mères célibataires noires affiliées au welfare rights movement choisirent de mettre en avant le service qu'elles rendaient à la société en élevant leurs enfants (Nadasen P., 2011: 56-59). Dans la seconde moitié du xx siècle, les Américaines jouèrent un rôle crucial tant dans la critique d'une vision trop étriquée des rapports liant l'individu au corps social que dans l'élaboration d'une alternative.

\section{Un enjeu pour l'histoire du genre et des femmes}

13 Dans les années 1960 et 1970, les travaux les plus influents des débuts de l'histoire des femmes et du genre étaient nombreux à porter sur la lutte pour le suffrage féminin. Ils contribuèrent à enraciner une chronologie et une mythologie de ce que l'ont appelait la première "vague » féministe. Dans ce récit, 1848 - la convention pour les droits des femmes de Seneca Falls - et 1920 - la ratification du $19^{\mathrm{e}}$ amendement - constituent deux bornes essentielles, récemment remises en question.

14 La décennie 1970 est caractérisée par une autre tendance de fond, l'intérêt pour le milieu féminin ouvrier et pour les rapports des ouvrières aux structures syndicales. Les féministes marxistes transposaient à leur champ d'étude les préoccupations de l'histoire sociale, mais les outils théoriques ne permettaient pas encore d'étudier les ramifications politiques des revendications économiques formulées par les femmes. Le concept de citoyenneté économique permet à présent de délimiter des objets d'étude nouveaux, telles que des formes d'activité politique particulières, ouvertes même aux Américains de la marge. D'un point de vue plus abstrait, une définition économique de la citoyenneté permet d'en apprendre plus sur l'implication des Américaines dans les institutions et la vie locales. Les derniers travaux sur l'économie informelle, elle-même un objet d'étude récent, ont montré le rôle central des commerçantes dans le tissu économique et social de la ville du xIX ${ }^{e}$ siècle (Luskey B. et W. Woloson, 2015).

Ces travaux nous amènent à repenser la république des producteurs rêvée par Jefferson. Des ouvrières du Gilded Age aux figures de proue féminines du mouvement pour les droits civiques - que l'étude des revendications d'ordre économique a d'ailleurs redéfini en early civil rights movement - de nombreuses activistes affirmèrent le caractère consubstantiel de l'économique et du politique. Les femmes noires en particulier maintinrent vivace la conscience économique de leur communauté tout au long du $\mathrm{xx}^{\mathrm{e}}$ siècle. L'évaluation du rôle central qu'elles jouèrent dans les levées de fonds 
nécessaires aux organisations civiques africaines-américaines fait partie des derniers développements de l'étude de la citoyenneté des femmes aux Etats-Unis.

\section{Conclusion}

Mettre ces femmes en lumière, c'est révéler les continuités. C'est aussi nuancer l'idée $\mathrm{du} 19^{\mathrm{e}}$ amendement vu comme un aboutissement et une fin. Avant et après 1920, des consommatrices citoyennes et engagées œuvraient au sein d'organismes nationaux comme la National Consumers' League (NCL) - et locaux - par exemple les associations de quartier dans lesquelles des femmes au foyer juives initièrent boycotts des bouchers et grèves des loyers. Par leurs activités, pas nécessairement tendues vers l'obtention du suffrage, ces femmes se voyaient, s'affirmaient et étaient vues au moins par certains comme des citoyennes avant tout.

\section{BIBLIOGRAPHIE}

Daune-Richard, Anne-Marie, « Homme, femme, individualité et citoyenneté », Recherches Féministes; Québec, vol. 21, n 1, 2008, p. 39-50. https://doi.org/10.7202/018307ar.

Guillen, Ana M., « Measuring Economic Citizenship : A Comment on Alice Kessler-Harris. » Social Politics : International Studies in Gender, State and Society, vol. 10, n² 2, 2003, p. 186-195. https:// www.muse.jhu.edu/article/50380.

Glickman, Lawrence, Buying Power: A History of Consumer Activism in America, Chicago, University of Chicago Press, 2009.

Isenberg, Nancy, Sex and Citizenship in Antebellum America, Chapel Hill, N.C., University of North Carolina Press, 1998.

Jacobs, Meg, Pocketbook Politics: Economic Citizenship in Twentieth-Century America, Princeton, Princeton University Press, 2005.

Jones, Martha S., « For Black Women, the 19th Amendment Marked Not the End, but the Beginning of the Movement For Voting Rights ", The Washington Post, 25 août 2020, https:// www.washingtonpost.com/opinions/2020/08/25/black-women-19th-amendment-didnt-endstarted-movement-voting-rights/. Page consultée le 31 août 2020.

Luskey, Brian P., et Wendy A. Woloson, eds., Capitalism by Gaslight : Illuminating the Economy of Nineteenth-Century America, Philadelphia, University of Pennsylvania Press, 2015.

Marshall, T. H., Citizenship and Social Class and Other Essays, Cambridge, Cambridge University Press, 1950.

Nadasen, Premilla, Rethinking the Welfare Rights Movement, New York, Routledge, 2011.

Vapnek, Lara, Breadwinners: Working Women and Economic Independence, 1865-1920, Urbana, University of Illinois Press, 2009. 
AUTEUR

JEANNE BOITEUX

Université Sorbonne Nouvelle 